\title{
A curious effect with reversed letters explained by a theory of schema
}

\author{
UTA FRITH \\ MRC Developmental Psychology Unit, Drayton House, Gordon Street, London, W.C.1., England
}

\begin{abstract}
It takes longer to find a normal $\mathrm{N}$ embedded in a context of reversed $\mathrm{Ns}$ than it takes to find a reversed $N$ embedded in a context of normal Ns. This is true for other letters as well and can be explained by a theory of schema. This theory holds that there is a schema for the normal form of a letter based on past experience, and that this schema is not a static but a flexible construct which is sensitive to the immediate cognitive context. This property of the schema enables recognition even if the letter is presented in a deviating form. A deviation, such as mirror reversal, may at times be as acceptable as the normal form of the letter. This is the case when context stimuli in a scanning task consist of reversed letters. If the target stimulus is the normal form of the letter, it is more difficult to detect since both context and target are accepted as the same letter.
\end{abstract}

A curious phenomenon was observed in a scanning task with normal and mirror reversed letters. Given the material in Fig. 1, it takes approximately twice as long to find normal Ns among reversed Ns (lower row) as it takes to find reversed Ns among normal Ns (upper row). Why should the lower row be more difficult than the upper row, when the only difference is the interchange between target and context shapes?

The notion of schemata provides a useful framework for considering this phenomenon. In the literature, the term "schema" seems to be used in at least two rather different senses. As introduced by Bartlett (1932) and further developed by Oldfield (1954), schemata are constructions that govern perception and memory. Neisser (1967) interprets Bartlett's schemata as frameworks into which specific representations can be fitted and sees a similarity to Bruner's (1957) coding systems. Gibson (1969), on the other hand, treats schemata as the representations themselves. In this paper also, schemata are seen as representations of stimuli in memory. This term, rather than "image," is used because it does not imply a static template or trace, but implies a construct that can change according to context requirements.

Evidence for the existence of schemata is particularly striking when their negative effects, such as biases in perception or distortions in memory, are studied. Much of this work is reviewed by Neisser (1967). Frith (1971, 1974) reported some evidence that there are schemata for familiar letters. Such schemata resulted in a bias in errors obtained in copying and matching tasks: a reversed letter was sometimes copied or matched as if it were a normal letter, but not vice versa. Such a bias was not found in preschool children unfamiliar with letters, nor was it found in adults when unfamiliar letterlike shapes were used.

It is known that simple figures such as letters can be recognized despite changes in their orientation, even though their normal orientation in space is preferred
(Rock \& Heimer, 1957). Even mirror-reversed letters can be recognized (Kolers, Eden, \& Boyer, 1964) without too much difficulty. By presenting letters in many different spatial orientations, Kolers (1968) demonstrated that a large variety of transformations of text can be read. It is also known that minor distortions in typescripts and handwriting, such as varying degrees of tilt, occur commonly and appear to matter little. Thus, it seems that different degrees of deviation from the ideal form of a letter may be accepted in different situations. Such an ability could be explained by assuming that there are schemata for familiar shapes that vary in relation to the immediate cognitive context. In this sense, recognition of familiar but distorted shapes can be said to depend not only on internal representations, built up in the past, but also on the stimulus environment at the time of recognition.

The following experiments demonstrate some effects which seem difficult to account for except by a theory of schema. The method used in the experiments is a variant of visual search which enables one to use time scores as indicators of underlying cognitive processes.

\section{EXPERIMENT I}

The reversed context effect illustrated in Fig. 1 was first found in a paper-and-pencil scanning task. A stimulus array consisted of 24 items in a row. Each item

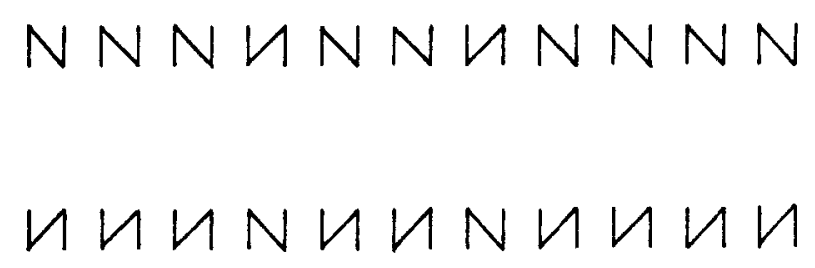

Fig. 1. 
Table 1

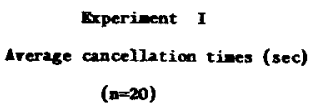

\begin{tabular}{|c|c|c|c|c|}
\hline Contert & Target & Time & $\mathbf{t}$ & px \\
\hline$N$ & K & 4.8 & \multirow{2}{*}{3.87} & \multirow[b]{2}{*}{.001} \\
\hline$M$ & $N$ & 10.2 & & \\
\hline$Z$ & $\Sigma$ & 3.4 & \multirow{2}{*}{2.03} & \multirow{2}{*}{.05} \\
\hline$S$ & $Z$ & 4.2 & & \\
\hline K & H & 4.4 & \multirow[b]{2}{*}{0.04} & \multirow{2}{*}{ n.s. } \\
\hline$\#$ & K & 4.4 & & \\
\hline$\not \neq$ & † & 3.5 & \multirow[b]{2}{*}{1.98} & \multirow[b]{2}{*}{ n.s. } \\
\hline$\neq$ & $\neq$ & 4.2 & & \\
\hline$V$ & $V$ & 4.6 & \multirow{2}{*}{0.57} & \multirow{2}{*}{ a.s. } \\
\hline$V$ & $V$ & 4.3 & & \\
\hline$\angle$ & $\triangle$ & 3.0 & \multirow{2}{*}{1.69} & \multirow[b]{2}{*}{ a.s. } \\
\hline$\Gamma$ & 7 & 3.3 & & \\
\hline
\end{tabular}

was drawn within a space of $6 \mathrm{sq} \mathrm{mm}$ and was spaced $6 \mathrm{~mm}$ apart from the next item. There were four targets, which were placed randomly within the row with the constraint that they should never be adjacent to each other and that they should not occur as the first or last item. Target and context stimuli were always mirror images of each other, and no other shapes were included in an array. For each array, there was a corresponding array with target and context stimuli interchanged, keeping the position of the targets constant. The stimuli shown in Table 1 were used to make up 12 such arrays.

The 12 arrays were presented in random order, differently for each S. Performance on each array was timed with a stopwatch, and timing started with the presentation of the array and stopped with the cancellation of the fourth target.

There were $20 \mathrm{Ss}$, mostly students. They were asked to scan through a row and to cancel the four "odd ones out" as fast as possible. The targets were never shown beforehand, but Ss were told that the first item in each row was context. To eliminate omission errors, they were also told that there would always be four targets. There were four pretraining trials using the stimulus pairs + and $x$, and $I$ and - . During these, it was emphasized that Ss should attend to "orientation" as the critical feature distinguishing targets from context.

The results are shown in Table 1 . The reversed context effect was found for the letters $\mathrm{N}$ and $\mathrm{Z}$ but was not found with arrays using unrecognizable distortions of these letters, namely and $\neq$. Nor was it found when components of the letters $\mathrm{N}$ and $\mathrm{Z}$ were used, such as $\mathrm{V}$ and $L$.

These results rule out the possibility that the reversed context effect could be due to simple shape characteristics, and indicate that is must be due to the fact that real letters are involved.

\section{EXPERIMENT II}

Scanning through many reversed, hence unfamiliar, letters may take longer just because the eye is arrested by the unusual more than by the usual. This hypothesis was tested by comparing reversed letter context and normal letter context with different letters as targets. Using the same procedure as before, Zs had to be found among reversed Ns and also among normal Ns. The same 20 Ss who took part in the first experiment needed 2.95 and $2.88 \mathrm{sec}$, on average, to cancel the targets in both conditions. The difference was negligible $(t=0.65)$. The same result was obtained for arrays with the letter $\mathrm{N}$ as target and the letter $\mathrm{Z}$ as context. Cancelling Ns in a context of reversed $\mathrm{Zs}$ took $2.87 \mathrm{sec}$, while cancelling Ns in a context of normal $\mathrm{Zs}$ took $3.10 \mathrm{sec}$ on average. This difference, which is in the opposite direction from that predicted by the hypothesis, was again not significant $(t$ $=1.68$ ). This experiment showed that the phenomenon does not just depend on a reversed letter context but depends on the fact that mirror images of the same letter are used for context and target.

\section{EXPERIMENT III}

It was now necessary to see if the same effect would be shown by other letters. Difficulties of interpretation arise with letters whose mirror-image forms are themselves real letters. These are the letters $b, d, p$, and q. For people who are thoroughly familiar with the alphabet, the mirror-reversed forms of these letters have separate identities. It is known to printers that these letters are particularly difficult to identify in reversed type (Jennet, 1964). As a reversed b becomes a d and a reversed $p$ becomes a $q$, there is no reversed letter context, only normal letter context. Therefore, one would expect no difference between scanning a row of bs for ds and scanning a row of ds for bs.

The experiment was carried out with 12 Ss. The method was identical to that used before. Table 2 shows the letters used. It also shows the average cancellation times and compares the two types of context. The reversed context effect appeared in every comparison, but was not equally strong in all the letters. That the effect should differ in strength is not unexpected. It is possible for Ss to disregard the fact that an array consists of letters of the alphabet. Particularly with the letter Q, most Ss said that they only looked at the detail of the diagonal line crossing the $\mathrm{O}$ to identify targets. With the letter $S$, the reversed form used resembled the number 2 . This may have prevented some Ss from seeing it as a reversed letter. Since the effect only occurs with letters, such strategies would vitiate the experiment. However, overall, reversed letter context resulted in significantly 
longer cancellation times than normal letter context. As predicted, the letters $b, d, p$, and $q$, for which there is no reversed letter context, did not show the effect. Cancelling ps among qs was as fast as cancelling qs among ps; this was true for $b$ and $d$ as well. We can, therefore, conclude that the reversed context effect applies to any letter which has a true mirror image.

\section{EXPERIMENT IV}

Having established that the effect is not specific to a particular letter, it is necessary to establish whether it is specific to particular experimental conditions. In Experiments I to III, four targets and 20 context stimuli were always used. One might ask whether varying the ratio of target to context stimuli would alter the effect. If the effect were due entirely to the nature of the target, then varying the ratio should make no difference. If however, the effect were due to the nature of the array as a whole, then altering the ratio should be critical.

The following experiment was carried out with eight new Ss. The letter $\mathbf{N}$ in its normal and reversed form was chosen as the stimulus throughout, since this letter had shown the reversed context effect particularly clearly. Eight arrays were presented which consisted as before of 24 elements in a row but now contained $8,12,16$, and 20 targets. This implies that there were sometimes more target than context stimuli in an array. It was therefore no longer possible to ask Ss to cancel the "odd ones out." Thus, before each trial, the Ss were shown the target and asked to find identical forms in the array. The procedure was otherwise the same as before. To eliminate omission errors, Ss were again told how many targets there were. The eight arrays were presented to each $\mathbf{S}$ in a balanced order, and this was preceded by several pretraining trials. The results are shown in Table 3. For 8 and 12 targets, the reversed context effect was again obtained, as in each case 7 out of the 8 Ss had longer

Table 2

Experiment III: Average Cancellation Times in Seconds

\begin{tabular}{ccccc}
\hline & \multicolumn{2}{c}{ Letter Context } & & \\
\cline { 2 - 3 } Letter & Normal & Reversed & $\mathrm{t}$ & $\mathrm{p}<$ \\
\hline $\mathrm{h}$ & 4.0 & 5.6 & 4.87 & .001 \\
$\mathrm{~L}$ & 3.3 & 4.4 & 3.60 & .005 \\
$\mathrm{y}$ & 3.4 & 4.4 & 3.08 & .01 \\
$\mathrm{R}$ & 4.2 & 6.5 & 2.14 & .05 \\
$\mathrm{C}$ & 3.5 & 4.8 & 2.08 & .05 \\
$\mathrm{~S}$ & 4.6 & 5.8 & 1.28 & n.s. \\
$\mathrm{D}$ & 4.1 & 5.3 & 0.84 & n.s. \\
$\mathrm{Q}$ & 5.5 & 5.9 & 0.47 & n.s. \\
& Normal Letter Context & & \\
& First & Second & & \\
& Letter & Letter & & \\
b d & 4.5 & 3.8 & 1.65 & n.s. \\
p q & 3.8 & 4.1 & 0.65 & n.s. \\
\hline
\end{tabular}

Note $-N=12$
Table 3

Experiment IV: Average Cancellation Times in Seconds

\begin{tabular}{|c|c|c|c|c|}
\hline \multirow[b]{3}{*}{$\begin{array}{l}\text { Mo. of } \\
\text { Context } \\
\text { Btimill }\end{array}$} & \multirow[b]{3}{*}{$\begin{array}{l}\text { No. of } \\
\text { Tareot } \\
\text { Stimild }\end{array}$} & & Context Targot & Context rarcet \\
\hline & & & $N M$ & $M N$ \\
\hline & & $\mathbf{n}$ & & \\
\hline 20 & 4 & 20 & 4.8 & 10.2 \\
\hline 16 & 8 & 8 & 8.9 & 12.1 \\
\hline 12 & 12 & 8 & 11.4 & 15.2 \\
\hline 8 & 16 & 8 & 12.1 & 12.3 \\
\hline 4 & 20 & 8 & 11.4 & 11.2 \\
\hline
\end{tabular}

cancellation times for normal Ns than for reversed Ns. Although significant on a sign test $(p=.035)$, the mean differences did not approach the magnitude of the difference previously obtained with four targets. For 16 and 20 targets, the mean cancellation times were nearly identical for the two comparison tasks. Thus, as soon as the number of targets exceeded half the stimuli in the array, the effect was no longer obtained. In this case, the nature of the task has changed drastically. Targets, by necessity, occur next to each other and may form long runs. Some Ss reported that they experienced a figure ground reversal, insofar as they apparently had to cancel ground stimuli and pass over figure stimuli.

Comparisons between arrays with different numbers of targets are not valid as the proportion of visual search and motor cancellation time changes. Comparisons between arrays with the same number of targets show that the ratio of target to context is a critical variable for the reversed context effect. This indicates that the effect cannot be explained in terms of the target alone, but that it must be explained in terms of the array as a whole.

The results also indicate that despite a major change in procedure, i.e., showing the target beforehand, the reversed context effect could still be elicited. Another indication of the reliability of the reversed context effect is its persistence with repetition. In the course of the series of experiments, two Ss had been used consistently in pilot trials, and it was found that despite extensive practice, the effect could always be obtained. As with many optical illusions, repeated exposure and knowledge of the effect does not seem to attenuate its strength.

\section{DISCUSSION}

The reversed context effect is surprising on two counts. Firstly, in the two tasks being compared, the discriminability of the target shapes is identical since the same pair of shapes is used in both cases. From this one would expect the two tasks to be of equal difficulty. This was not found to be true except for extremely high target/context ratios. Secondly, the effect implies that it is more difficult to search for familiar, normally oriented letters than for unfamiliar, reversed letters. This is contrary to the expectation that familiar targets may be labeled more readily and hence cancelled more quickly. 
A closer analysis of the task may show why neither of these plausible expectations were fulfilled. Finding few targets embedded in rows of many identical stimuli involves spotting deviations from a norm established by these context stimuli. Recognizing a reversed letter involves spotting a deviation from a norm established by past experience with the correct form of the letter. This norm is internally represented and constitutes the schema. When scanning for a reversed letter among normal letters, the two norms coincide, that is, the reversed letter target is a deviation from the immediate context of normally oriented letters as well as a deviation from the schema. On the other hand, when scanning for a normal letter in an array of reversed letters, the two norms are in conflict: the normally oriented form of the letter is a deviation from the norm established by the context while it is not a deviation in terms of the schema. The slowing down of scanning time might be a result of this conflict.

This only applies if there are few targets and hence these can be readily treated as "odd ones out," i.e., deviations from the context. When there are more target than context stimuli, then effectively the context stimuli have become the "odd ones out." It is possible that some Ss would therefore consider the task as one of searching for the context stimuli, which are the only ones not to be cancelled. Such a strategy reverses the nature of the task. Therefore, when targets exceed context stimuli, the reversed context effect cannot be reliably observed since there is no longer proper control over which of the two shapes the $S$ treats as target. This might explain why the effect disappeared with high target/context ratios.

It remains to be explained why scanning is slowed down when a norm established by the immediate context and a norm established by the schema are in conflict. For the efficient performance of the scanning task, the schema of the letter used should be irrelevant since only the norm provided by the context is necessary for defining the target. However, the reversed context effect demonstrates that when familiar letters are involved, the internal schema intrudes. It can be hypothesized that the particular nature of this intrusion is a result of the sensitivity of the schema to context. It has been suggested in the introduction that in order to handle the variable forms that letters may take, the criteria for letter identification are constantly altered by the context in which that letter occurs. In the scanning task, this alteration will depend on the context stimuli. When the context stimuli are normal letters, strict acceptance criteria can be applied, since these letters are presumably close to the schema. When context stimuli are reversed letters, this context creates a norm that is different from the ideal form based on the letter in its normal orientation. In order to include this immediate context norm, the acceptance criteria relating to the schema must be lowered. Thus, reversed letters become as acceptable as normally oriented letters and can be "read" easily. For the scanning task, this implies that normal letter targets now fall into the same category as reversed letters and thus become less distinct. Hence, the $\mathrm{S}$ experiences greater difficulty in identifying targets. This then may explain why it takes longer to cancel few normal letters in a context of many reversed letters.

In the present task, context sensitivity of the schema is associated with negative effects. However, in real life the effect will more often be positive. Context sensitivity of the schema implies that distorted objects are turned automatically into recognizable. familiar objects. This is a highly efficient procedure especially for reading, since print and handwriting are characterized by countless deviations from some ideal alphabet. It is difficult to imagine that this feat is achieved other than by means of schemata.

\section{REFERENCES}

Bartlett, F, Remembering. Cambridge: University Press, 1932.

Bruner, $J$. $S$. Going beyond the information given. In Contemporary approaches to cognition. A symposium held at the University of Colorado. Cambridge, Massachusetts: Harvard University Press, 1957.

Frith, U. Why do children reverse letters? British Journal of Psychology, 1971,62, 459 .

Frith, U. In ternal schemata for letters in good and bad readers. British Journal of Psychology, 1974, 65, 233-241.

Gibson, E. J. Principles of perceptual learning and development. New York: Appleton-Century-Crofts, 1969.

Jennet, S. The making of books. London: Faber, 1964. P. 53.

Kolers, P. A. The recognition of geometrically transformed text. Perception \& Psychophysics, 1968, 3, 57-64.

Kolers, P. A., Eden, M., \& Boyer, A. Reading as a perceptual skill, M.I.T. Research Laboratory of Electronics Quarterly Progress Report, 1964, 74, 214-217.

Neisser, U. Cognitive psychology. New York: Appleton-Century-Crofts, 1967.

Oldfield, R. C. Memory mechanisms and the theory of schemata. British Journal of Psychology, 1954, 45, 14-23.

Rock, I., \& Heimer, W. The effect of retinal and phenomenal orientation on the perception of form. American Journal of Psychology, 1957, 70, 493-511.

(Received for publication September 14, 1973; revision received March 15, 1974.) 